\title{
Proof of principle for the generation of a transplantable recellularized liver graft
}

T

he ability to manufacture auxiliary liver grafts for transplantation may now be a step closer thanks to the work of Korkut Uygun and colleagues.

The shortage of donor livers for transplantation is an ongoing issue and ways to alleviate the shortage are being sought. One alternative is hepatocyte transplantation, but the efficacy of this approach is currently limited by cell supply and low engraftment efficiency. Tissue engineering also has potential as an alternative treatment option. However, although work in animal models has shown that cell engraftment is improved by tissue engineering, this technique also has its limitations.

"One of the major issues in tissue engineering is to come up with a scaffold that mimics the organ's microarchitecture and its extracellular matrix composition," explains Uygun. He and his team therefore decided to investigate whether a transplantable liver graft could be generated by the decelullarization and recellularization of a whole liver, thus taking advantage of the existing scaffold.

The authors were encouraged by work demonstrating the potential of decellularization for the engineering of several different tissues-esophagus, trachea, artery, bladder, skin and heartand the potential to use organs deemed unsuitable for transplantation as a source for decellularized liver matrix (DLM).

The initial step that Uygun et al. took was to perfuse ischemic rat livers through the portal vein with an anionic detergent to lyse the cells present and solubilize components of the cell cytoplasm. After $72 \mathrm{~h}$ they were left with the DLM-a translucent liver-shaped scaffold that was free of nuclei, cytoplasm and cells, but retained components of extracellular matrix similar to native liver. The retention of a functional vascular bed in the DLM was confirmed by various methods (portal vein dye perfusion, corrosion casting and scanning electron microscopy), which
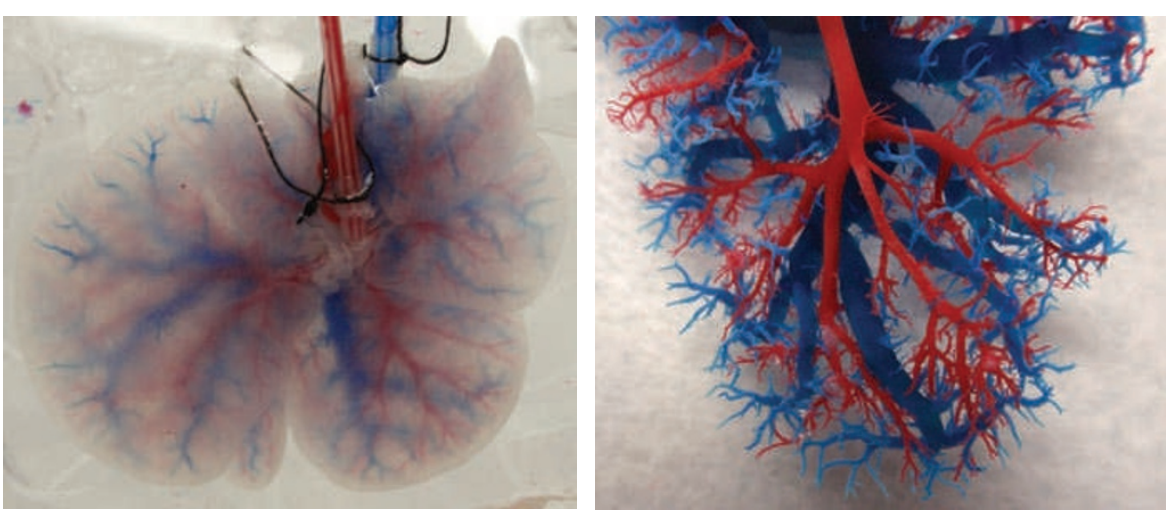

The vascular bed is intact and functional in the decellularized liver matrix. The portal (red) and venous (blue) vasculature are visible in the translucent matrix (left) and in a corrosion cast model (right). Courtesy of K. Uygun, with permission from NPG.

suggests that it would be possible to achieve physiologic flow through the graft.

Next the authors recellularized the DLM via an in vitro perfusion-seeding technique. Approximately 50 million primary rat hepatocytes were perfused into the DLM through the portal vein in a four-step seeding procedure (12.5 million cells per step). The recellularized liver grafts then underwent in vitro culture in a specially designed perfusion chamber.

Histologic analysis revealed that the hepatocyes engrafted around large vessels at $4 \mathrm{~h}$, but were distributed throughout the parenchyma at later time points ( 1 day, 2 days and 5 days). In addition, there was minimal cell death during the 5-day perfusion period. Immunostaining confirmed that the functional characteristics of the engrafted hepatocytes were similar to those in native liver. The engrafted hepatocytes were also metabolically active, as determined by the measurement of albumin production and urea synthesis. The expression of genes encoding various drug metabolizing enzymes was also confirmed.

Uygun and colleagues then tested the scalability of their recellularization method. As nonparenchymal cells will be an essential part of any reconstructed liver graft, they attempted to seed the recellularized graft with endothelial cells. Their attempt was successful and the viability of the engrafted hepatocytes maintained. They also wanted to see whether the DLM could be seeded with 200 million hepatocyes ( $20 \%$ of native rat liver hepatic mass and more than double the minimum hepatic mass required for therapy). This seeding was also successful and the perfusion-culture system shown to cope with the additional cell numbers.

In the final stage of the study, recellularized grafts were transplanted into rats that had undergone a unilateral nephrectomy-the grafts were connected to the renal vein and artery. After $8 \mathrm{~h}$ in vivo the grafts were harvested and the hepatocytes shown to have suffered minimal damage. Hepatocyte morphology, position and function had also been maintained. Similar, positive results were also achieved during $24 \mathrm{~h}$ ex vivo blood perfusion of recellularized grafts.

In light of their progress, Uygun is cautiously optimistic for the future, "Hopefully we have established the basics for liver tissue engineering and we and others can take it further."

Natalie J. Wood

Original article Uygun, B. E. et al. Organ reengineering through development of a transplantable recellularized liver graft using decellularized liver matrix. Nat. Med. doi: $10.1038 / \mathrm{nm} .2170$ 